\title{
Priority-Based Hybrid MAC Protocol for VANET with UAV-Enabled Roadside Units
}

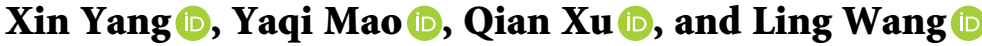 \\ School of Electronics and Information, Northwestern Polytechnical University, Xi'an 710072, China \\ Correspondence should be addressed to Xin Yang; xinyang@nwpu.edu.cn
}

Received 19 November 2021; Revised 14 December 2021; Accepted 19 January 2022; Published 4 February 2022

Academic Editor: Guangwei Yang

Copyright (c) 2022 Xin Yang et al. This is an open access article distributed under the Creative Commons Attribution License, which permits unrestricted use, distribution, and reproduction in any medium, provided the original work is properly cited.

\begin{abstract}
Vehicular ad hoc networks (VANETs) connect vehicles with other network units through wireless communication, thus improving traffic efficiency and safety by interacting with traffic information in networks. Roadside unit (RSU) plays an important role in VANETs in connecting vehicles with the transportation centre. With the rapid development of unmanned aerial vehicles (UAV), UAV-enabled RSU (U-RSU) is an effective way to promote the performance of VANETs. In this paper, a novel medium access control (MAC) layer protocol named PHB-MAC is proposed based on the priority setting of transmitted messages. Firstly, the priority-based contention mechanism is presented to ensure the transmission efficiency of the safety message. Then, the proposed MAC protocol combining contention and adaptive scheduled scheme is described in detail. Finally, the numerical analysis and simulation results of PHB-MAC and other existing protocols are presented. It is revealed that the proposed protocol improves throughput by at least $30 \%$. Meanwhile, it reduces packets drop rate and delay by $21 \%$ and $22 \%$, respectively. Finally, the numerical analysis and simulation results are given to prove the advancement of the proposed protocol on throughput, packets drop rate (PDR), and delay.
\end{abstract}

\section{Introduction}

In recent years, the rapid increase of vehicles around the world has brought about more serious challenges to traffic safety and efficiency. With the rapid development of wireless communication, sensing, mobile computing, and automatic control technology, the vehicular ad hoc network (VANET) has emerged to enhance traffic safety and improve traffic efficiency [1]. VANET is a specific ad hoc network that is composed of many dynamic nodes without any centralized control equipment. In VANET, the vehicle communicates with other vehicles by onboard unit (OBU). Also, vehicles equipped with OBU can build a transmission link with roadside unit (RSU), which is fixed to the side of the road. RSU can communicate with OBUs in its transmission range, including relay and providing traffic information [2]. In this way, the vehicle obtains real-time status information of surrounding vehicles by VANET to avoid road accidents and enhance traffic safety. In addition, with the widespread application of unmanned aerial vehicles (UAV) in the construction of smart cities, UAV-enabled RSU (U-RSU) is an important way to enhance the performance of VANET. Compared with fixed RSU, U-RSU has the advantages of flexibility, scalability, and connectivity. In addition, U-RSUs can provide effective help to fix RSUs in the case of serious traffic congestion. Unlike other fixed wireless networks, the nodes in VANET are fast-moving vehicles; the primary service of VANET is a safety service [3]. As a result, the key features and performance requirements of VANET are concluded as follows:

(i) The nodes in VANET show a high mobility characteristic.

(ii) The nodes in VANET are vehicles with communication capabilities; unlike traditional fixed wireless networks, they have the characteristics of high mobility as fast as $120 \mathrm{~km} / \mathrm{h}$, and the relative speed can achieve more than $200 \mathrm{~km} / \mathrm{h}$. Under the circumstances, the VANET needs to handle the 
problem of fast and frequent network accessing and switching of vehicles [4].

(iii) The network topology changes frequently.

(iv) Because of the fast-moving of large numbers of vehicles, the topology of VANET changes frequently. In this way, the transmission links between nodes are unstable, which leads to a failed transmission and influences the reliability of networks. What is more, the design of medium access control (MAC) and routing protocol in VANET is more complicated than normal wireless networks [5].

(v) The quality of service (QoS) requirement of VANET is specific.

(vi) The main task of VANET is providing safety services to all vehicles and enhancing traffic safety. Therefore, low latency and packet drop rate (PDR) are both crucial performances to VANET. Besides, high throughput is another important requirement to maintain information transmission in networks. In this way, not only can data information be delivered efficiently, but also the safety message can be transmitted reliably [6].

In the last decade, various MAC protocols were proposed to improve the performance of VANET. In general, these protocols can be divided into three categories, which are contention-based protocols, contention-free protocols, and hybrid protocols, respectively. Contention-based protocols are mainly based on the ALOHA or carrier sense multiple access (CSMA) mechanism, in which there is no predetermined allocation of transmission resources [7]. If the channel is sensed as idle by a node, it accesses the channel and starts transmission directly. Otherwise, if the channel is busy, the node has to pick up a backoff value from the contention window $(\mathrm{CW})$ and start a countdown. When the backoff value reaches 0 , the transmission begins. If there is another collision, a larger backoff value is selected randomly. The circulation of backoff does not finish until the transmission is successfully completed. The IEEE 802.11p MAC protocol is a typical protocol based on the CSMA mechanism; like many other contention-based protocols, the QoS cannot meet the performance requirement in high-density VANET. Meanwhile, many contention-free MAC protocols are proposed to improve QoS and efficiency of VANET, especially in a high-density application scenario [8]. Network resources are scheduled in VANET before access and transmission. The main mechanism of the contention-free protocol is based on time division multiple access (TDMA), frequency division multiple access (FDMA), and some other multiple access methods. In this transmission scheme, each node has its own time slot or frequency band to access channel and transmit information [9]. However, when the transmission load of VANET is low, many nodes still occupy the time slots or frequency bands without transmission tasks. In this way, network resources are wasted and lead to a decline in efficiency. To make a tradeoff between contentionbased and contention-free protocols, hybrid protocols have been proposed in recent years. The research of hybrid mechanisms focuses on the TDMA-CSMA hybrid protocol to enhance traffic safety and improve transmission efficiency. The difficulty and challenge of the hybrid protocol is how to design specific protocols according to different application scenarios [10]. What is more, the existing MAC protocols seldom make full use of both TDMA and CSMA period to meet the performance requirements in highdensity and fast-moving VANET.

In this paper, a novel hybrid MAC protocol named PHBMAC (priority-based hybrid MAC layer protocol) is proposed for VANET to enhance the transmission efficiency of data information and promote the reliability of safety message transmission. The main contributions of this paper are concluded as follows:

(i) The proposed hybrid protocol is based contending and scheduled hybrid mechanism. Nodes in networks reserve their own transmission slot by a priority-based grouping contention scheme in PHB-MAC. Then, according to the reserved time slots, nodes transmit data in their own slots to avoid contention.

(ii) In the proposed grouping contention scheme, nodes access channels by groups are employed instead of all nodes contending for access. In this way, both efficiency and access fairness are improved simultaneously. In addition, due to the priority setting of PHB-MAC, high-priority information is guaranteed to be timely delivered in its own groups.

(iii) In the adaptive TDMA-based period of the proposed protocol, time slots are allocated not only to transmission between OBUs and U-RSU but also to the information exchanges between adjacent U-RSUs. The trajectory of the vehicle is along the road and predictable, so it is efficient for a U-RSU to transmit information of vehicles in its transmission range to the next U-RSU with which the vehicle will communicate.

(iv) The performance of PHB-MAC on throughput, PDR, and delay is analyzed and simulated in this paper. Simulation results are presented to prove that the PHB-MAC can significantly promote the efficiency and traffic safety of VANET, especially in a high-density scenario.

The rest of this paper is organized as follows. Section 2 gives a brief review of MAC protocol design in VANETs. Next, Section 3 describes the proposed PHB-MAC protocol in detail. Section 4 presents a theoretical analysis of throughput, PDR, and delay of PHB-MAC. Then, the performance evaluation of PHB-MAC in VANET is presented in Section 5. Finally, Section 6 concludes the paper.

\section{Related Works}

Normally, MAC layer protocols for wireless networks are classified into three types based on access modes, namely, contention-based, contention-free, and hybrid, respectively. 
Therefore, a brief and concise review of different types of MAC protocols is described in this section.

In contention-based MAC protocols, the IEEE $802.11 \mathrm{p}$ scheme is widely used in VANET, which is derived from IEEE 802.11 scheme and based on the carrier sense multiple access/collision avoidance (CSMA/CA) mechanism [11]. The channel division and modulation method are optimized for VANET in IEEE 802.11p. In CSMA/CA-based VANET, nodes in networks contend for transmission after a channel sensing period. As soon as the channel is sensed as idle, nodes start to access the channel after a distributed interframe spacing (DIFS). If the channel switches to busy and collision happens, the node turns to backoff and contends again after a contention window (CW). Each idle slot passes, and the CW counter is reduced by 1 . When CW returns to 0 , the transmission starts again. In addition, each time a collision happens, the CW increases. The CSMA/CA-based IEEE $802.11 \mathrm{p}$ protocol has the characteristics of easy implementation and distribution without a central control node. However, its performance seriously deteriorates in high-density networks. What is more, the safety service of VANET cannot be guaranteed in IEEE $802.11 \mathrm{p}$ protocol. A vehicle-to-vehicle protocol named EDF-CSMA is proposed based on IEEE 802.11p to guarantee the QoS of the networks [12]. EDF-CSMA dynamically adjusts the priority of realtime streaming to avoid collision and introduces an admission control policy according to time constraints to provide guaranteed QoS in multichannel environments. But there is still the problem of heavy collisions in high-density VANET when nodes enter and leave the network frequently. In addition, a mobile edge computing- (MEC)- based CSMA mechanism MAC protocol is proposed to promote the performance of VANET [13]. The MEC-based protocol analyzes the average uplink local delay of a vehicle driving on a highway for sending a packet to its serving edge node along the highway. This MEC-based protocol focuses on transmission delay; however, special requirements for safety services are not taken into account.

Also, many contention-free MAC protocols are designed based on a contention-free mechanism, especially on the TDMA scheme. In TDMA, time-domain resources are divided into successive frames. In each frame, nodes access and occupy one or more time slots for transmission as scheduled. TDMA can effectively reduce transmission collision in high-density VANET and enhance efficiency. However, as a scheduled protocol, it will lead to transmission resource waste if a node does not have data to transmit in its own time slot. A prediction-based TDMA MAC protocol named PTMAC is proposed to decrease the number of packet collisions [14]. It is demonstrated to be suitable for VANET in an urban area with unbalanced traffic densities. But in low-density VANET, the predication process can lead to more overhead and increase the delay, especially for safety message delivery. As a result, the QoS of VANET decreases. Meanwhile, another TDMA-based MAC protocol called MoMAC is proposed in [15]. In MoMAC accessing algorithm, the time slot resources are adaptively divided into many subsets according to the road topology. In addition, each node broadcasts safety messages together with the time slot occupying information of neighbors. It improves efficiency and reduces data collision in high-density VANET, but the collision of safety messages is still a problem to solve.

Hybrid MAC protocols combine the advantages of contention-based and contention-free protocols to improve the performance of VANET. A hybrid MAC protocol named HER-MAC is proposed in [16]. The control channel transmission is divided into reserved period and contention period. It allows nodes to transmit a safety message on the control channel and transmit a nonsafety message on the service channel during the interval of the control channel. In this way, the efficiency of transmission is improved. However, there are too many overhead packets that need to be transmitted in HER-MAC, which also increases the collision rate. To solve the problems above with HER-MAC, a hybrid TDMA/CSMA multichannel MAC protocol is presented [17]. It can also enhance broadcasting efficiency and improve the throughput of the control channel by removing unnecessary overhead packets. But the priority of safety message transmission cannot be guaranteed, thus affecting the QoS of VANET. Meanwhile, thanks to the rapid development of fourth-generation $(4 \mathrm{G})$ mobile communication technology, VMaSC-LTE is proposed, combining IEEE 802.11p-based multihop clustering and the $4 \mathrm{G}$ cellular mechanism [18]. VMaSC-LTE decreases the number of cluster heads and increases the stability of networks, therefore improving efficiency. The clustering algorithm in VMaSC-LTE is easily affected by the changes in node density in VANET, which leads to a waste of channel resources in low-density networks.

As a result, fully considering the application performance requirements of VANET with UAV-enabled RSU, the characteristics of hybrid MAC protocols, and the shortcomings of existing protocols, this study proposes a high efficiency contending and adaptive TDMA hybrid MAC protocol for VANET to improve throughput, delay, and PDR.

\section{PHB-MAC Protocol Design}

In this section, details of the proposed PHB-MAC protocol for VANET are described. The network under consideration consists of vehicles running two-way on the road. Vehicles are all equipped with OBUs, including global position system (GPS) receivers. Through the OBU, vehicles can communicate with U-RSUs.

3.1. Assumptions. Some assumptions are made for the proposed protocol in VANET as follows:

(1) Every vehicle is equipped with a GPS device and knows its own real-time state information, such as location, speed, and moving direction. This information is broadcast by OBUs at each frame of the group that it belongs to.

(2) The slot information of U-RSU is kept by all vehicles in its transmission range and its one-hop U-RSUs transmission range. 


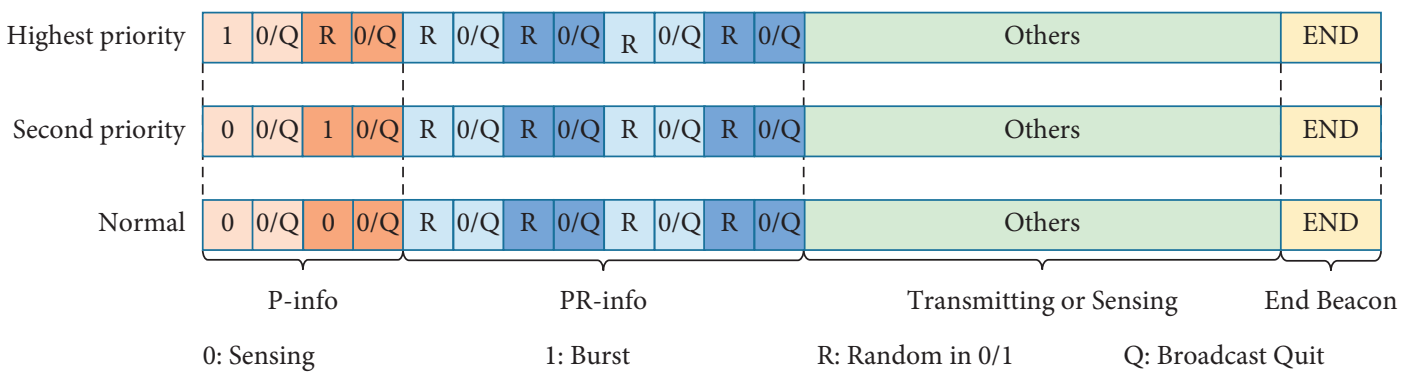

FIgURE 1: Designing different priority information transmission.

(3) For a newly joined vehicle, it needs to listen to the channel and wait until the next frame starts for its transmission.

(4) The road information, such as road length, traffic congestion, and the number of vehicles, is available for OBUs.

3.2. Message Priority Setting. Designing a transmission scheme according to the priorities of messages is a significantly effective way to enhance transmission performance and traffic safety in VANET. Therefore, it is necessary to set message priority before transmission. In the proposed protocol, transmitted messages are divided into three priorities. First and foremost, safety message is set as the highest priority since it is crucial for all vehicles to run safely. Then, control messages and some messages about important vehicle status information are defined as the second priority. Finally, other messages are set to normal priority. In addition, the ratio of different priority packets is set as $80: 16: 4$ (normal: second priority: highest priority), which is based on the $80 / 20$ rule.

To ensure that high-priority messages are transmitted successfully, as described in Figure 1, priority information (P-info) and priority random information (PR-info) are set before the transmission period. For easy understanding, it is defined that 0 is denoted as sensing, 1 means burst transmission, $R$ is randomly selected from 0 to 1 , and $Q$ means the OBU fails in contention and broadcast a Quit beacon. In the proposed protocol, each slot contains two parts in the contention period. The first part is used for contention; OBUs sent burst or keep silent and sense the channel randomly. In the second part, if the OBU fails in contention, it broadcasts a Quit beacon. Other OBUs keep the sense and listen to the beacon. In case an OBU does not sense anything in the second parts of the slot, it wins contention.

As the rules above, to make sure the highest priority message is transmitted as soon as possible, it is defined that the first slot in P-info of the highest priority is $1-0 / Q$. At the same time, if there are some other OBUs that want to transmit a second priority or normal message, they will sense the burst transmission of the highest priority message in the first slot. So, they quit the contention and sense the channel for the next transmitting opportunity. In this way, the highest priority message can be sure to win the contention. Furthermore, if there is no highest priority message to transmit in the first slot, all OBUs sense the channel. Then, the OBU, which wants to transmit a second priority message, starts a burst in the second slot. Other OBUs that want to send a normal message sense the burst and quit the contention. Otherwise, if all the messages to be sent are normal, the OBUs start the PR-info period to contend for transmitting. Finally, the OBU that wins the contention begins transmitting data to U-RSU in the transmitting period; other OBUs sense the channel until they receive the END beacon and start the next cycle. In addition, the number of slots in the PR-info period is not a constant quantity. It adapts to the degree of traffic congestion and updates every cycle. To describe the message transmission scheme more clearly, the message priority setting and contention processing are presented in Figure 2.

For example, in Figure 3, four OBUs contending to transmit normal messages are described to show the contention process in the same priority. In the $\mathrm{P}$-info period, all of them sense the channel to verify whether there is any higher priority message need to be transmitted. Then, in the PR-info period, every OBU generates a set of random numbers in $0 / 1$ to fill the first part in each slot. The second part of each slot is determined by the first part. In the first part, the random number is 0 , and the OBUs switch to a sensing state. If it senses others' burst, which means it fails in contention, the OBU broadcasts Quit and quits the contention. Otherwise, it switches to a sense or burst state according to the corresponding number in the generated random sequence. In the illustration, it is assumed that the generated random sequence of OBU 2 is a 4 -bit number 1110, which fills the first part in each slot. As a result, other OBUs failed in the contention because they all sense the burst transmission from OBU 2 and thus quit contention. Meanwhile, the 8-bit contending sequence of OBU 2 is acquired as 10101000. When OBU 2 finishes transmitting data, it broadcasts an END beacon. Then, OBU 4 senses the END beacon and starts transmitting. Generally, as the priority setting above in one contention group, if an OBU senses $n$ Quit beacons in the quit and sensing period, it will begin its transmission period when the $(n+1)$ th sensed END beacon finishes. In addition, the slot allocated to the contending sequence is adaptively adjusted to fit the traffic. For each priority level, a collision happens in the contending slot, which means the length of the contending slot is not enough. The U-RSU will increase the length of the next contending time slot by 1 in the corresponding priority level. On the contrary, if all OBUs finish contending with extra contending slots left, the U-RSU will reduce the length of the 


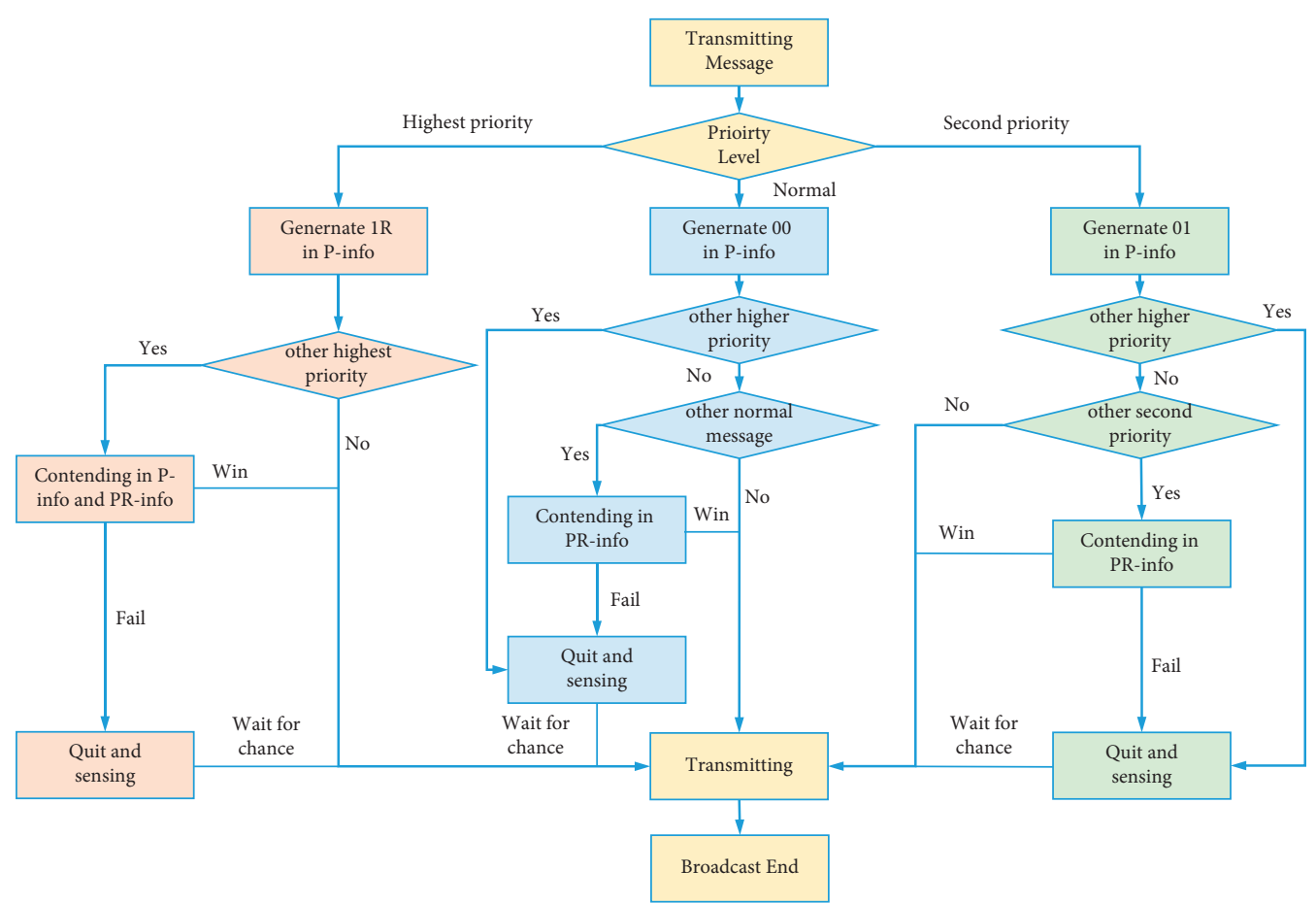

FIgure 2: Message priority setting and contention processing.

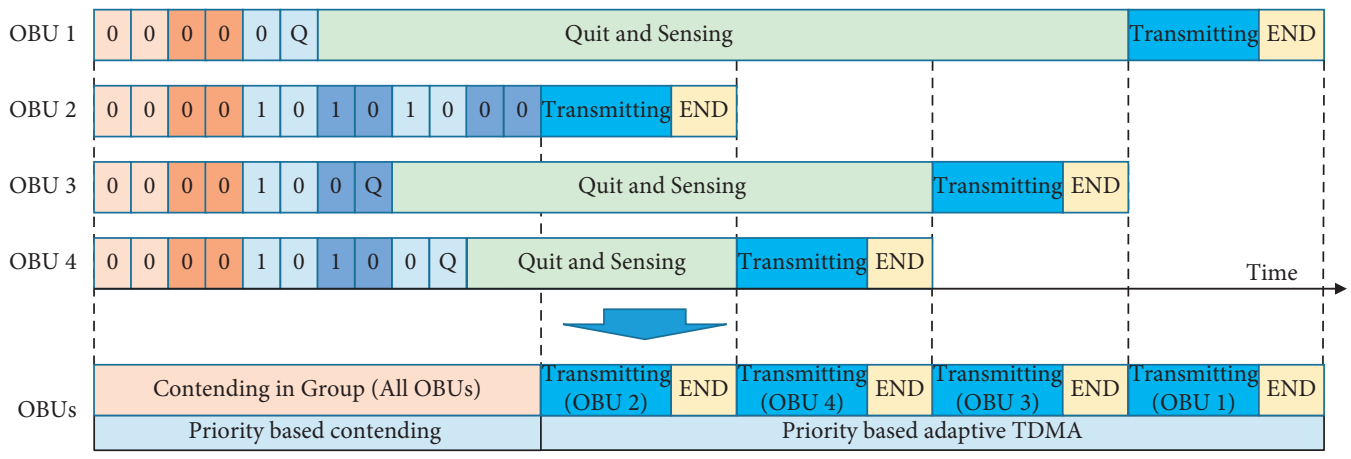

FIGURE 3: Group contending in OBU-to-OBU transmission.

next contending time slot by 1 in the corresponding priority level. Specifically, the variation range of the contending slot is confined to integers in $[1, C L x-m]$, where $C L x-m$ means the maximum value of $C L$ (contending slot length). In this paper, the length of contending slot for highest priority, second priority, and normal is defined as positive integer $C L h, C L s$, and CLn, respectively, which are defined as CLh$m \geq C L h \geq 1, C L s-m \geq C L s \geq 1$, and CLn-m $\geq C L n \geq 1$ in the protocol.

3.3. Frame Design of PHB-MAC. According to the proposed assumptions and the definition of message priority, the frame design of the proposed protocol is described in this section. To explain the protocol process more clearly, the general scenario of VANET is presented in Figure 4. Assume that all vehicles are equipped with OBUs and drive on the right side of the road.
U-RSUs are distributed along the road to communicate with OBUs in their transmission range. The distance between each two U-RSUs must be less than the maximum transmission distance of U-RSU. In addition, if an OBU is within the transmission range of two adjacent U-RSUs at the same time, it will select the closer U-RSU for transmission. In the proposed protocol, define that all OBUs communicating with U-RSU-A and U-RSU-B make up group A and group $B$, respectively. Other groups are defined in the same way. To reduce transmission collision, each group is divided into $n$ subgroups for accessing and transmission separately. That is, the area covered by a group is divided into Ns equal parts; all vehicles in each part form a subgroup. For example, Figure 4 illustrates the subgroups distribution of group A and group $\mathrm{B}$.

To describe the frame design of PHB-MAC in detail, the transmission of U-RSU-A, U-RSU-B, and OBUs in A1 and OBUs in $\mathrm{B} 1$ is illustrated in Figure 5 as a one-slot example. 


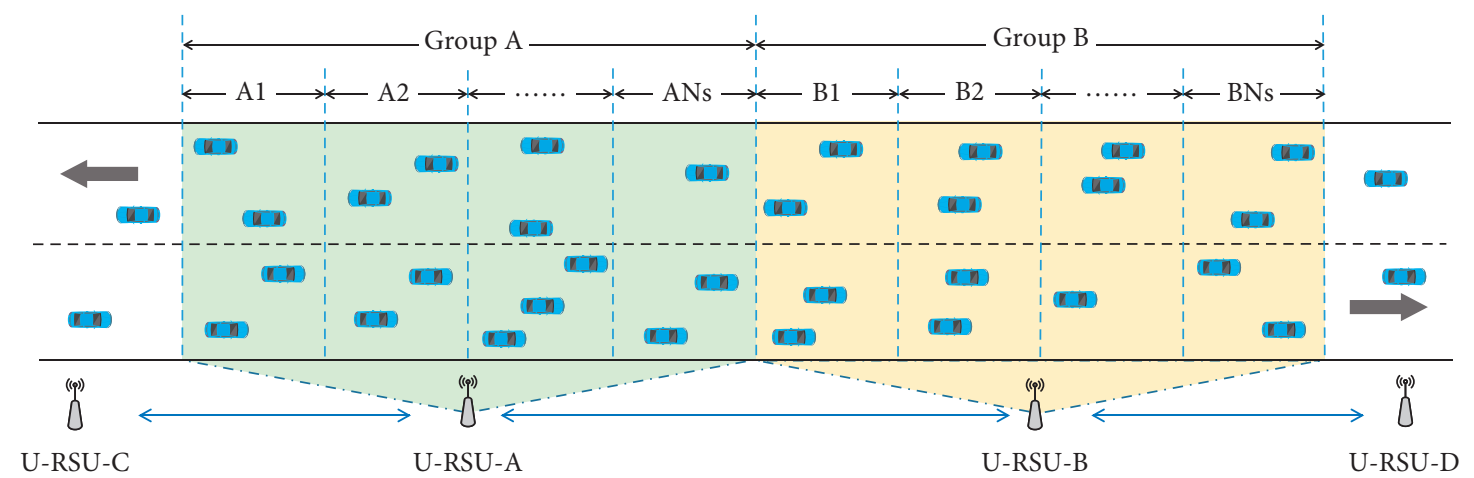

FIgURE 4: General scenario of VANET and subgroups distribution.

\begin{tabular}{|c|c|c|c|c|c|c|}
\hline \multirow{3}{*}{ OBUs in $\mathrm{B} 1$} & \multirow{2}{*}{ Update } & \multirow{2}{*}{\multicolumn{2}{|c|}{ Priority based contending }} & \begin{tabular}{l|l|} 
OBU1 in B1 & OBU2 in B1 \\
\end{tabular} & OBUn in B1 & \multirow{2}{*}{$\begin{array}{c}\text { OBUs in } \\
\mathrm{B} 2, \mathrm{~B} 3, \ldots, \mathrm{BNs}\end{array}$} \\
\hline & & & & \multicolumn{2}{|c|}{ Priority based adaptive TDMA } & \\
\hline & $\uparrow$ & & & $\begin{array}{l}\uparrow \mid \\
\mid \downarrow\end{array}$ & $\begin{array}{l}\uparrow \mid \\
\mid \downarrow\end{array}$ & \\
\hline \multirow{3}{*}{ U-RSU-B } & Control & with U-RSU-A & with U-RSU-D & \multirow{2}{*}{\multicolumn{2}{|c|}{ OBUs-RSU Transmission in Group B }} & \\
\hline & Messages & RSU to RSU & ransmission & & & \\
\hline & & $\begin{array}{l}\uparrow \mid \\
\mid \downarrow\end{array}$ & & & & \\
\hline \multirow{3}{*}{ U-RSU-A } & Control & with U-RSU-B & with U-RSU-C & \multirow{2}{*}{\multicolumn{2}{|c|}{ OBUs-RSU Transmission in Group A }} & \\
\hline & Messages & RSU to RSU & ransmission & & & \\
\hline & $\downarrow$ & & & $\begin{array}{lll}\uparrow & 1 \\
1 & \downarrow\end{array}$ & $\begin{array}{ll}\uparrow & \\
\mid \downarrow & \downarrow\end{array}$ & \\
\hline \multirow{2}{*}{ OBUs in $\mathrm{A} 1$} & Update & \multirow{2}{*}{\multicolumn{2}{|c|}{ Priority based contending }} & \begin{tabular}{|l|l|} 
OBU1 in A1 & OBU2 in $\mathrm{Al}$ \\
\end{tabular} & OBUn in $\mathrm{Al}$ & OBUs in \\
\hline & & & & Priority based a & & $\mathrm{A} 2, \mathrm{~A} 3, \ldots, \mathrm{ANs}$ \\
\hline
\end{tabular}

Figure 5: Example of transmission using PHB-MAC.

When the slot begins, RSU-A and U-RSU-B broadcast control messages to OBUs in $\mathrm{A} 1$ and $\mathrm{B} 1$, respectively. According to the control message, OBUs know when and what states they will switch to in this slot. Meanwhile, OBUs update their own state information as soon as receiving the control message. The next is the priority-based contending period; OBUs in a subgroup A1 or B1 contend based on the priority of messages to reserve transmission in the adaptive TDMA period. The detailed process of the priority-based contending period is shown in Figure 3. At the same time, to take full use of the time slot, U-RSU-A and U-RSU-B both communicate with their adjacent U-RSUs. In this way, key information on road conditions and vehicles is timely transmitted between two groups. The length of time slot for RSU-to-RSU transmission is set equal to the priority-based contending period, in which the transmitting time spent on left U-RSU and right U-RSU is half and half. Then comes to OBUs-RSU transmission period of each group, which follows the priority-based adaptive TDMA scheme in Figure 3. Specifically, in the transmission period between OBUs in A1 and U-RSU-A, all OBUs in A1 communicate with U-RSU-A in their own time slot as the scheduled order of prioritybased contending period. When the last OBU finishes communication with U-RSU and broadcasts the END beacon, the mission of the current subgroup is over. The next OBU subgroups start transmission tasks by receiving control messages from U-RSU and updating their own state information. Then, the process is repeated again as the previous subgroup.

\section{Performance Analysis}

Based on the assumptions and descriptions of the proposed protocol above, the performance analysis is presented in this section by establishing a Markov model, as shown in Figure 6. The symbols used in the analysis of the proposed protocol are summarized in Table 1.

In the analysis of the proposed protocol, $P_{c}$ denotes the collision probability of the transmission, $P_{s}$ is the success probability of packet transmission, $P_{t r}$ denotes the probability that there is a packet to be sent, and the maximum contending slot length of $x$ priority message in the $i$ th slot is defined as $C L_{x-m i}$, where $x$ represents a kind of message among highest, second, or normal priority. Therefore, the one-step transmission probabilities and steady-state probabilities of the Markov model are calculated as follows:

$$
\begin{gathered}
P\{i, k \mid i, k\}=1-P_{c}, \quad\left(1 \leq k \leq \mathrm{CL}_{n-\mathrm{mi}}, 1 \leq i \leq n\right), \\
P\{i, k+1 \mid i, k\}=P_{c}, \quad\left(1 \leq k \leq \mathrm{CL}_{n-\mathrm{mi}}, 1 \leq i \leq n\right), \\
P\{i, k \mid i-1, k\}=\frac{1-P_{s}}{C L_{x-m n}}, \quad\left(1 \leq k \leq \mathrm{CL}_{x-\mathrm{mi}}, 1 \leq i \leq n\right),
\end{gathered}
$$




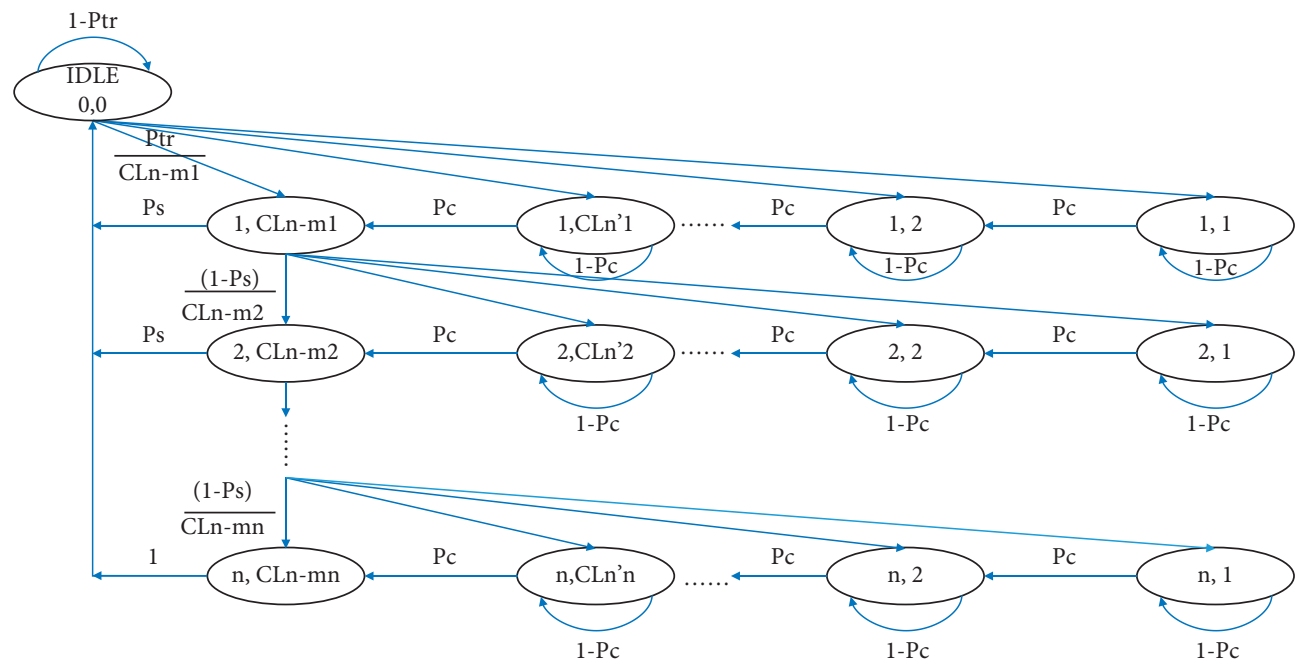

Figure 6: Markov model for PHB-MAC protocol.

TABLE 1: Symbols summary for model analysis.

\begin{tabular}{lc}
\hline Parameters & Description \\
\hline $\mathrm{N}$ & The number of time slots in priority-based adaptive TDMA period. \\
$P_{c}$ & The collision probability of transmission. \\
$P_{s}$ & The success probability of packet transmission. \\
$P_{\mathrm{tr}}$ & The probability that there is a packet to be sent. \\
$P_{\mathrm{bus}}$ & The probability that the channel is busy. \\
$\tau_{\mathrm{pac}}$ & The probability that a node transmits a data packet in an arbitrary time slot. \\
$N_{\text {cont }}$ & The number of nodes contending for transmission simultaneously. \\
$\mathrm{CL}_{x-\mathrm{mi}}$ & The maximum contending slot length of $x$ priority message in the $i$ th slot $(x=$ highest/second/normal). \\
$T_{\mathrm{OBU}-h}$ & The time cost on highest priority message transmission of OBU. \\
$T_{\mathrm{OBU}-s}$ & The time cost on second priority message transmission of OBU. \\
$T_{\mathrm{OBU}-n}$ & The time cost on normal message transmission of OBU. \\
$T_{\mathrm{ctrl}}$ & The time cost on control message transmission. \\
$T_{\text {contx }}$ & The time cost on contending of $x$ priority information. \\
$T_{\text {pac }-x}$ & The time cost on packet transmission of an $x$ priority message. \\
$T_{\mathrm{re}-x}$ & The time cost on retransmission of an $x$ priority message. \\
$S_{\mathrm{Total}}$ & The total throughput of the networks. \\
$S_{\mathrm{RR}}$ & The throughput of RSU-RSU transmission. \\
$S_{\mathrm{OR}}$ & The throughput of OBU-RSU transmission.
\end{tabular}

$$
\begin{gathered}
P\left\{0,0 \mid i, \mathrm{CL}_{x-\mathrm{mi}}\right\}= \begin{cases}P_{s},\left(1 \leq k \leq \mathrm{CL}_{x-\mathrm{mi}}, \quad 1 \leq i \leq n-1\right), \\
1,\left(1 \leq k \leq \mathrm{CL}_{x-\mathrm{mi}}, \quad i=n\right),\end{cases} \\
P\{1, k \mid 0,0\}=\frac{P_{\mathrm{tr}}}{\mathrm{CL}_{x-m 1}, \quad\left(1 \leq k \leq \mathrm{CL}_{x-m 1}\right),} \\
P\left\{1, k \mid i, \mathrm{CL}_{x-\mathrm{mi}}\right\}=\left\{\begin{array}{l}
\frac{P_{s} P_{\mathrm{tr}}}{\mathrm{CL}_{x-m 1}},\left(1 \leq k \leq \mathrm{CL}_{x-m 1}, \quad 1 \leq i \leq n-1\right), \\
\frac{P_{\mathrm{tr}}}{\mathrm{CL}_{x-m 1},},\left(1 \leq k \leq \mathrm{CL}_{x-m 1}, \quad i=n\right) .
\end{array}\right.
\end{gathered}
$$

It can be obtained by equations (1)-(5) that (i) When a message arrives at an idle node, it starts to transmit with a random CL in the first slot. Otherwise, the node continues to remain at an idle state.

(ii) Every time there is a transmission collision, $k$ increases by 1 until it reaches the maximum value $\mathrm{CL}_{x-\mathrm{mn}}$.

(iii) If the message is successfully transmitted or reaches its maximum retransmission $\mathrm{CL}_{x-\mathrm{mi}}$ in all $n$ time slots, the node will reset the CL value and turn to an idle state.

(iv) When a message is not successfully transmitted in the first contending time slot, it will continue to be transmitted in the next slot with a random value of CL. 
Let $b_{i, k}$ denote the stationary state probability of the state $\{i, k\}$. Based on the model, the probability that a node transmits a data packet in an arbitrary time slot is represented as

$$
\tau_{\mathrm{pac}}=\frac{1-\left(1-P_{s}\right)^{n}}{P_{\mathrm{tr}}} b_{1, \mathrm{CL} x-m 1} .
$$

Therefore, the busy probability of the channel can be obtained as

$$
P_{\text {bus }}=1-\left(1-\tau_{\text {pac }}\right)^{N_{\text {cont }}}
$$

where $N_{\text {cont }}$ means the number of nodes, which are contending for transmission simultaneously. From (7), the collision probability of the transmission is derived as

$$
P_{c}=1-N_{\text {cont }}\left(1-\tau_{\text {pck }}\right)^{N_{\text {cont }}{ }^{-1}} \text {. }
$$

Also, the success probability of packet transmission is expressed as

$$
P_{s}=N_{\text {cont }} \tau_{\text {pac }}\left(1-\tau_{\text {pac }}\right)^{N_{\text {cont }}-1} .
$$

Let $T_{\text {ctrl }}, T_{\text {cont }-x}, T_{\text {pac }-x}$, and $T_{\text {re- } x}$ be the duration for control message transmission, the duration for contending of $x$ priority message, the duration for packet transmission of $x$ priority message, and the duration for retransmission of $x$ priority message, where $x$ is one of high priority, second priority, and normal priority. As a result, the time cost on the highest, second, and normal priority message transmission of OBU is given as

$$
\begin{aligned}
& T_{\mathrm{OBU}-h}=T_{\text {ctrl }}+T_{\text {cont }-h}+T_{\mathrm{pac}-h}+T_{\mathrm{re}-h}, \\
& T_{\mathrm{OBU}-s}=T_{\text {ctrl }}+T_{\text {cont-s}}+T_{\mathrm{pac}-s}+T_{\text {re- } s}, \\
& T_{\mathrm{OBU}-n}=T_{\text {ctrl }}+T_{\text {cont }-n}+T_{\mathrm{pac}-n}+T_{\mathrm{re}-n} .
\end{aligned}
$$

In this paper, the throughput is defined as the ratio of the average transmitted payload length and the average duration in a slot time as follows:

$$
S=\frac{E[\text { transmitted payload length in a slot time }]}{E[\text { duration of a slot time }]} \text {. }
$$

Let $S_{\text {Total }}$ be the total throughput of the networks, which can be computed by

$$
S_{\text {Total }}=S_{\mathrm{RR}}+S_{\mathrm{OR}}
$$

where $S_{\mathrm{RR}}$ and $S_{\mathrm{OR}}$ are the throughput of RSU-RSU transmission and OBU-RSU transmission, respectively.

Let $L_{R_{K-1, K}}$ and $L_{R_{K, K+1}}$ be the length of the packet transmitted between two adjacent U-RSUs that is between the $(K-1)$ th U-RSU and the Kth U-RSU, or the Kth U-RSU and the $(K+1)$ th U-RSU. Hence, the throughput of RSURSU transmission is derived as

$$
S_{\mathrm{RR}}=\frac{L_{R_{K-1, K}}+L_{R_{K, K+1}}}{T_{R_{K-1, K}}+T_{R_{K, K+1}}}=\frac{L_{R_{K-1, K}}+L_{R_{K, K+1}}}{T_{\operatorname{cont} K}},
$$

where $T_{R_{K-1, K}}$ and $T_{R_{K, K+1}}$ represent the duration for transmission between the $(K-1)$ th U-RSU with the Kth U-RSU and the Kth U-RSU with the $(K+1)$ th U-RSU, respectively. In the proposed protocol, let $T_{\text {cont } K}$ be the duration for the contending period of the Kth U-RSU; we have $T_{R_{K-1, K}}+T_{R_{K, K+1}}=T_{\text {contK }}$ to support the derivation of (14).

The throughput of OBU-RSU transmission is made up of the throughput of the three-priority information. Let $S_{\mathrm{OhR}}$, $S_{\text {OsR }}$, and $S_{\text {OnR }}$ represent the three-priority information, which are highest, second, and normal priority, respectively. Hence, the throughput of OBU-RSU is obtained as

$S_{\mathrm{OR}}=S_{\mathrm{OhR}}+S_{\mathrm{OsR}}+S_{\mathrm{OnR}}=\frac{P_{\mathrm{trh}} P_{\mathrm{sh}} L_{h}+P_{\mathrm{trs}} P_{\mathrm{ss}} L_{s}+P_{\mathrm{trn}} P_{\mathrm{sn}} L_{n}}{T_{\mathrm{ctrl}}+T_{\mathrm{Tcont}}+T_{\mathrm{Tpac}}+T_{\mathrm{Tre}}}$,

where $T_{\text {Tcont }}$ means the total time of contending in OBURSU transmission.

Let $P_{\text {conth }}, P_{\text {conts }}$, and $P_{\text {contn }}$ denote the contending probability of the highest, second, and normal priority information, respectively. So $T_{\text {Tcont }}$ is given as

$$
T_{\text {Tcont }}=P_{\text {conth }} T_{\text {conth }}+P_{\text {conts }} T_{\text {conts }}+P_{\text {contn }} T_{\text {contn }} .
$$

Let PDR be the packet drop rate of the transmission, which means the probability that a packet will be dropped after the maximum retransmission limit. It is given as

$$
\operatorname{PDR}=\left(1-P_{s}\right)^{M_{\text {mre }}}
$$

where $M_{\text {mre }}$ is the maximum number of retransmissions for a packet.

In this paper, the delay is defined as the mean time spent on the successful transmission of information. If a packet is dropped, it can be regarded as an unsuccessful transmission. So, it will not be included in the evaluation of the transmission delay. As a result, the transmission delay is given as

$$
\begin{aligned}
\text { Delay }= & E\left[T_{\text {succ }}\right]=\left(1-P_{\text {tr }}\right) T_{\text {slot }}+P_{\text {tr }} P_{s}\left(T_{\text {ctrl }}+T_{\text {Tpac }}\right) \\
& +P_{\text {tr }}\left(1-P_{s}\right)\left(T_{\text {Tcont }}+T_{\text {Tre }}\right),
\end{aligned}
$$

where $T_{\text {succ }}$ is the time spent on successful transmission of information and $T_{\mathrm{Tpac}}$ and $T_{\mathrm{Tre}}$ are the time spent on packet transmission and retransmission, respectively.

\section{Simulation Results}

In this section, the proposed protocol PHB-MAC is compared with other protocols such as CB-MAC and NC-PNC MAC in the simulation of MATLAB. The simulation scenario is a 2-kilometer long two-way straight road with a number of moving vehicles. The U-RSUs are evenly arranged on the roadside with an interval of 500 meters. The average speed of each vehicle is defined as $60 \mathrm{~km} / \mathrm{h}$, with a range of $50 \mathrm{~km} / \mathrm{s}$ to $70 \mathrm{~km} / \mathrm{s}$. It is assumed that the data arrival follows Poisson distribution in the simulations of this paper. The distribution function of Poisson distribution is described as $P(X=k)=\lambda^{k} / k ! e^{-\lambda}$, where $k$ is the number of times that data arrived and $k=0,1,2 \ldots$

In 100 data packets of the simulations, we assume that there are 80 normal priority data packets, 16-second priority 
data packets, and 4 highest priority data packets. To simulate the application scenarios of the VANET more realistically, the ratio of different priority packets we set conforms to the $80 / 20$ rule. That is to say, 80 percent of the transmitted data packets belong to normal priority data packets. 80 percent of the remaining 20 percent are second priority data packets; others are highest priority data packets. The main parameters used in the simulations are summarized in Table 2.

Figure 7 presents that the throughput versus different numbers of vehicles varies from 10 to 150 . It is easy to find that as the number of vehicles grows, the throughput decreases obviously. Specifically, in Figure 7(a), the proposed PHB-MAC is compared with CB-MAC and NC-PNC MAC in the simulation. The packets arrival rate is set at 100 packets/s. When the number of vehicles is less than 50 , the throughput of all three protocols declined rapidly, but by similar values. As the number of vehicles is larger than 50 but less than 100, the throughput provided by CB-MAC is obviously less than the other two protocols. When the number of vehicles is larger than 100, PHB-MAC shows its advantage because the proposed contending and adaptive scheduled hybrid scheme can mitigate transmission collision. As there are 150 vehicles in the simulation, the proposed PHB-MAC can provide nearly $30 \%$ and $45 \%$ higher throughput than NC-PNC MAC and CB-MAC, respectively. In conclusion, PHB-MAC performs better than other protocols in the simulation, especially in dense networks.

Then, Figure $7(\mathrm{~b})$ presents the simulation results of throughput versus the number of vehicles with different packets arrival rates. The packets with three different priorities (highest, second, and normal priority) accounted for $80 \%, 16 \%$, and $4 \%$, respectively. Obviously, a lower packet arrival rate leads to a higher throughput due to the fewer transmission collisions in the networks. When the number of vehicles is more than 50 , the throughput of 20 packets/s is 2 to 4 times higher than others. However, high data arrival rates are of more concern in the practical application of VANET. In the simulation, we can find that as the data arrival rate increases from 100 packets/s to 500 packets/s, there is no serious drop in throughput (only $8 \%$ ). That is, the throughput of the proposed protocol does not degrade significantly when the network load increases in a heavy traffic situation. When the number of vehicles continuously grows, the throughput of 100 packets/s and 500 packets/s maintains at nearly $5.1 \mathrm{Mbps}$ and $4.6 \mathrm{Mbps}$, respectively. The main reason is that the priority-based adaptive TDMA scheme can relieve congestion effectively in heavy payload transmission.

In Figure 7(c), the throughput of safety messages versus the number of vehicles is simulated. The safety message is defined as the highest priority message in this paper, which has a close relationship with traffic safety. The throughput values of all protocols decrease with the increase of vehicles. However, PHB-MAC can mitigate throughput degradation due to its priority-based contending scheme. In this way, safety message transmission and traffic safety are guaranteed to the maximum extent. In contrast, the safety message throughput of NC-PNC MAC and CB-MAC reduces significantly as the vehicles increase. PHB-MAC improves
TABLE 2: Symbols summary in simulation.

\begin{tabular}{lc}
\hline Parameters & Value \\
\hline Carrier frequency & $2.4 \mathrm{GHz}$ \\
Length of the normal priority data packet & 1000 bytes \\
Length of the second priority data packet & 200 bytes \\
Length of the highest priority data packet & 50 bytes \\
Transmission range of OBU and U-RSU & $500 \mathrm{~m}$ \\
Length of the control message & $512 \mathrm{bits}$ \\
Length of the END beacon & $64 \mathrm{bits}$ \\
Slot duration & $50 \mathrm{~ms}$ \\
SIFS & $10 \mathrm{us}$ \\
DIFS & $50 \mathrm{us}$ \\
\hline
\end{tabular}

safety message throughput by almost $90 \%$ and $360 \%$ compared to the other two protocols.

Figure 8 shows the simulation results of PDR versus the number of vehicles. The PDR not only reflects the reliability of the protocols but also is an important performance indicator of the network. It can be observed that more vehicles on the road lead to a higher PDR because the transmission collision and congestion occur more frequently as vehicles increase. The PDR of different protocols is shown in Figure $8(\mathrm{a})$, with the packets arrival rate at 100 packets/s. When the number of vehicles is less than 40 , there is little difference in the PDR provided by each protocol. This means that there is not much difference in the reliability among all protocols when the network is not heavily loaded. However, how to reduce the PDR under high transmission load is the concern of the VANET. As the simulation results show, compared with CB-MAC and NC-PNC MAC, when the number of vehicles is greater than 60 , PHB-MAC can reduce the packet loss rate by almost $28 \%$ and $21 \%$, respectively. In other words, the proposed protocol can reduce PDR effectively in heavy transmission scenarios.

To evaluate the reliability performance of PHB-MAC, the PDR versus the number of vehicles at different packets arrival rates is simulated. Obviously, the higher the data arrival rate, the higher the packet drop rate. When the data arrival rate is 20 packets/s and 100 packets/s, the PDR stays at a relatively low value (less than $20 \%$ ) when the number of nodes is less than 80 . As the number of vehicles increases, the value of PDR increases sharply and reaches $48 \%$ (20 packets/s) and $88 \%$ (100 packets/s) at 150 vehicles eventually. Meanwhile, with the data arrival rate coming to 500 packets/s, the PDR increases rapidly as the number of vehicles is more than 30 . When there are 80 or more vehicles on the road in the simulation, the PDR reaches a relatively high level.

Also, the PDR versus the number of vehicles at safety message transmission is simulated by using different protocols. To provide a strong guarantee for traffic safety, the PDR of safety message is of great importance. On the whole, it is clear to find that the proposed PHB-MAC provides the best PDR among all of the protocols. To be specific, the values of PDR provided by all protocols are at the same level when there are no more than 50 vehicles in the networks. As the number of vehicles increases, the advantage of the proposed protocol on PDR becomes more and more 


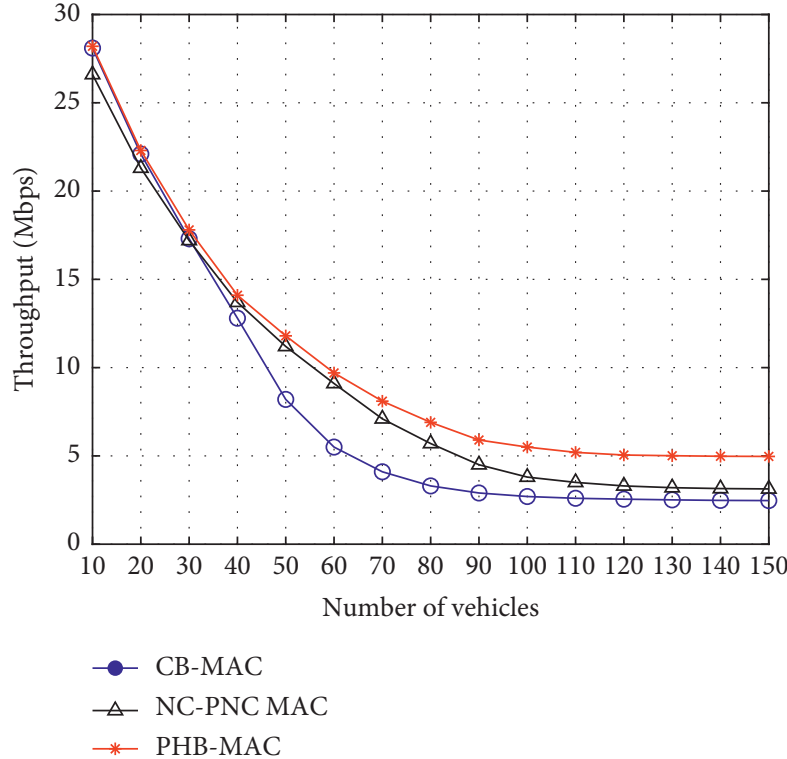

(a)



(b)

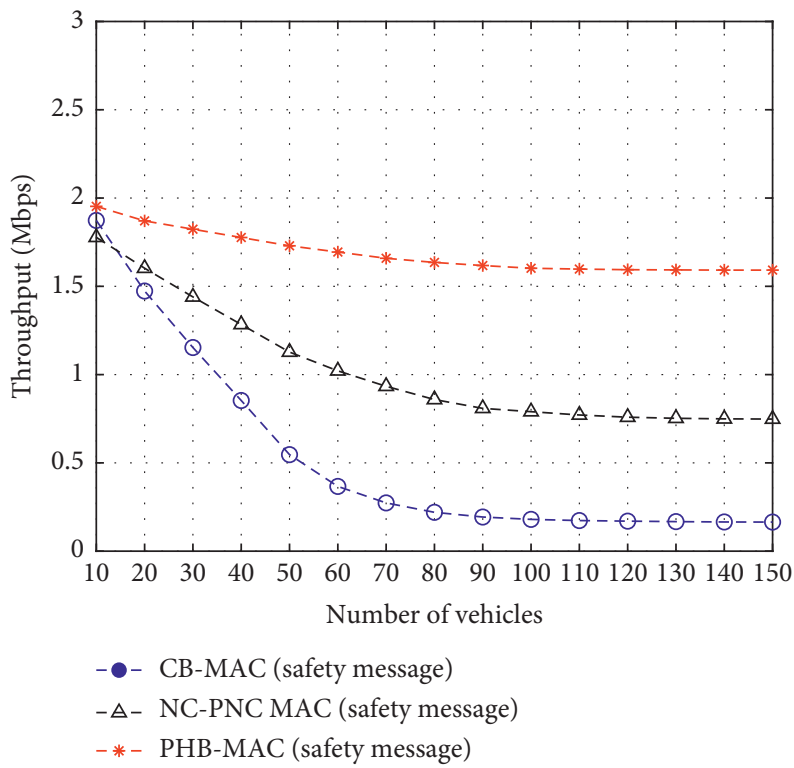

(c)

Figure 7: (a) Throughput versus the number of vehicles for different protocols. (b) Throughput versus the number of vehicles for different packets arrival rate. (c) Throughput versus the number of vehicles for safety message.

obvious. Compared to CB-MAC and NC-PNC MAC, PHBMAC reduces the $\mathrm{PDR}$ by as much as $81 \%$ and $72 \%$, respectively.

As a vital performance, the transmission delay of $\mathrm{CB}$ MAC, NC-PNC MAC, and PHB-MAC is simulated with the results shown in Figure 9 9(a). The packets arrival rate is set at 100 packets/s in the simulation. For all of the protocols, heavier traffic leads to a higher transmission delay. The value of delay shows a swift growth when the number of vehicles is less than 70. Meanwhile, the delay basically remains unchanged when there are more than 100 vehicles in the networks. In particular, when the number of vehicles is less than 40 , the three protocols can provide a similar delay performance. However, with the increase of vehicles, PHBMAC reveals its advantage because of the adaptive TDMA scheme. The time slot allocation for OBUs can be changed adaptively depending on traffic load to reduce the time cost of queuing and contending. In general, the proposed protocol can provide a $28 \%$ and $22 \%$ less delay than CB-MAC and NC-PNC MAC in the simulations, respectively.

Meanwhile, the delay of PHB-MAC is simulated under the condition of different packets arrival rates. Therefore, different packets arrival rates represent different traffic conditions. In heavy traffic situations, the frequent generation and forwarding of vehicle and road condition information result in a high packet arrival rate. The simulation 


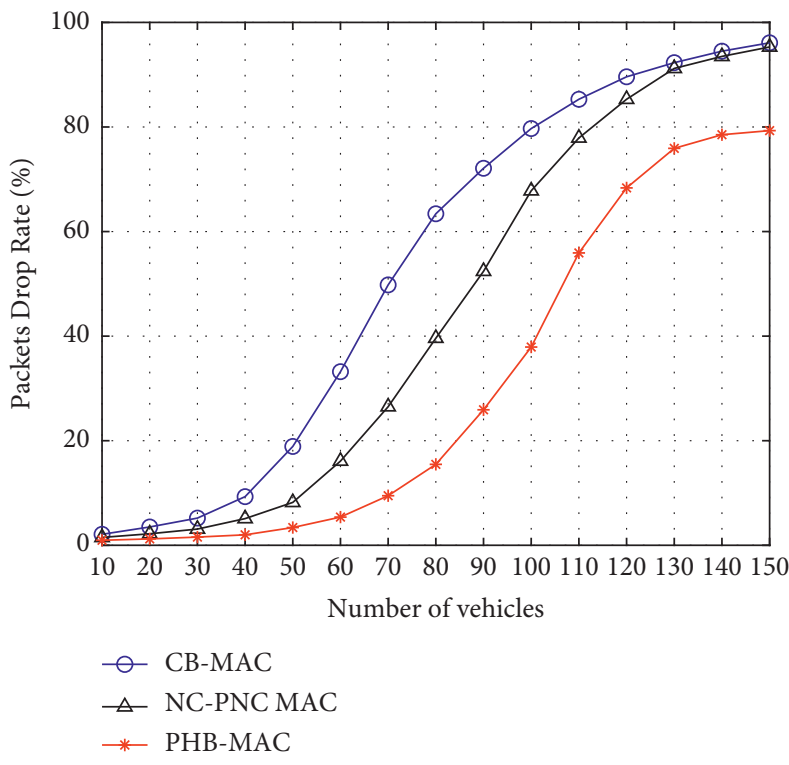

(a)



(b)

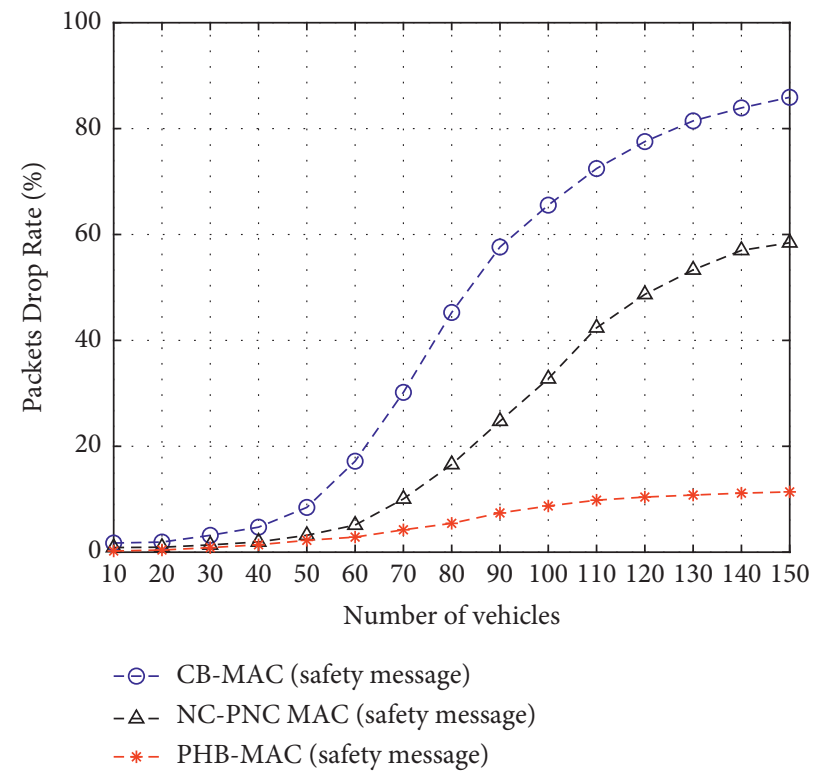

(c)

FIGURE 8: (a) PDR versus the number of vehicles for different protocols. (b) PDR versus the number of vehicles for different packets arrival rate. (c) PDR versus the number of vehicles for safety message.

results are shown in Figure 9(b). Obviously, a higher packets arrival rate leads to a significantly higher delay. Specifically, when there are 20 data packets arriving per second, the delay generally remains at a relatively low level. When there are 150 vehicles in the network, the maximum delay is still less than $20 \mathrm{~ms}$. As the packets arrival rate increases to 100 packets/s, the delay shows significant growth and finally arrives at nearly $41 \mathrm{~ms}$. In heavy traffic situations with 500 packets arriving per second, the delay rises rapidly when the number of vehicles is less than 50. However, PHB-MAC can effectively restrain the growth of delay on account of OBUs' subgrouping and adaptive TDMA scheme. The maximum delay arrives at nearly $68 \mathrm{~ms}$, which is acceptable in VANET.
Finally, the safety message transmission delay of the three protocols is simulated, and the results are shown in Figure 9(c). In case there are no more than 50 vehicles in the networks, the delay provided by PHB-MAC and NC-PNC MAC is similar, whereas the delay of CB-MAC increases sharply with the increase of vehicles. When the number of vehicles is larger than 70, the delay of CB-MAC and PHBMAC maintains steady. But the delay of NC-PNC MAC still grows rapidly. Compared with $\mathrm{CB}-\mathrm{MAC}$ and NC-PNC MAC in large-scale VANET, PHB-MAC can reduce the delay by almost $38 \%$ and $29 \%$, respectively. As a whole, PHB-MAC reveals a prominent advantage in the performance of delay compared to the other two protocols. 


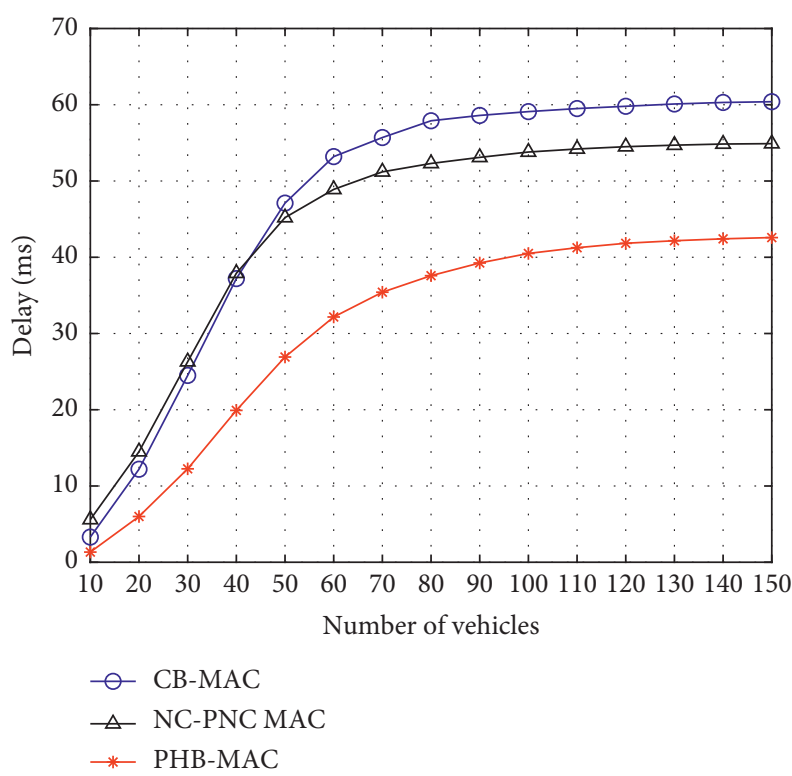

(a)

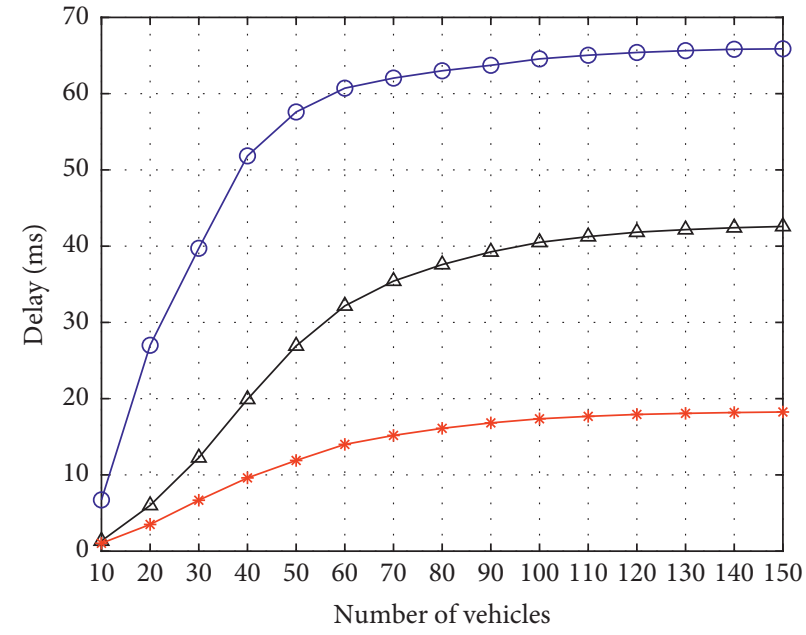

○- 500 packets/s

$\triangle 100$ packets/s

* 20 packets/s

(b)

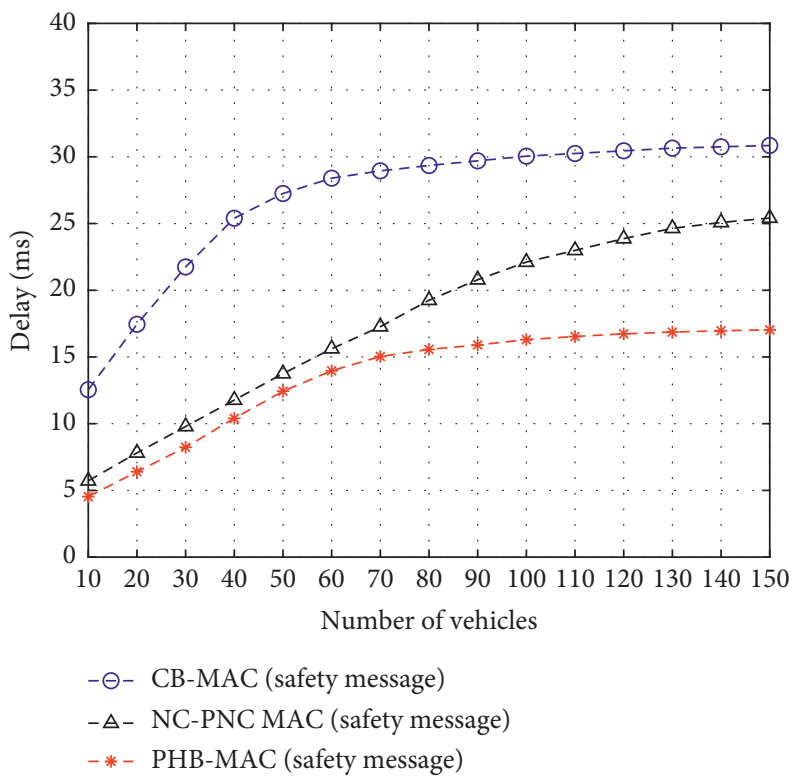

(c)

FiguRE 9: (a) Delay versus the number of vehicles for different protocols. (b) Delay versus the number of vehicles for different packets arrival rate. (c) Delay versus the number of vehicles for safety message.

\section{Conclusions}

In this paper, a hybrid MAC layer protocol based on information priority is proposed for enhancing the transmission performance of VANET with UAV-enabled RSU. In particular, the proposed PHB-MAC protocol significantly promotes the efficient and reliable transmission of the safety message. First of all, all transmitted messages are divided into three categories based on the message priority setting. The fundamental purpose is to ensure that the safety message can be efficiently transmitted, which is critical to traffic safety. Secondly, a hybrid MAC layer-accessing scheme including RSU-to-RSU and OBU-to-RSU transmission link is proposed. In the RSU-to-RSU transmission slot, OBUs in its U-RSU's range are contending based on priority. In addition, the results of the contention can make guidance for the design of the coming adaptive TDMA period. Thirdly, the performance of PHB-MAC on throughput, PDR, and delay is simulated. The simulation results illustrate the advantage of the proposed protocol in VANET, especially for safety message transmission.

\section{Data Availability}

The data used to support this study are included in the paper or available from the corresponding author upon request. 


\section{Conflicts of Interest}

The authors declare that there are no conflicts of interest regarding the publication of this paper.

\section{Acknowledgments}

This work was supported by the National Natural Science Foundation of China under Grants nos. 61901390 and 61901382 and the Natural Science Basic Research Plan in Shaanxi Province of China under Grant no. 2020JQ-201.

\section{References}

[1] M. Hadded, P. Muhlethaler, A. Laouiti, R. Zagrouba, and L. A. Saidane, "TDMA-based MAC protocols for vehicular ad hoc networks: a survey, qualitative analysis, and open research issues," IEEE Communications Surveys \& Tutorials, vol. 17, no. 4, pp. 2461-2492, 2015,

[2] A. U. Haq and K. Liu, "Review of TDMA-based MAC protocols for vehicular ad hoc networks," in Proceedings of the IEEE 18th International Conference on Communication Technology (ICCT), pp. 459-467, Chongqing, China, October 2018.

[3] S. Ansari, M. Sanchez, T. Boutaleb, S. Sinanovic, C. Gamio, and I. Krikidis, "SAI: Safety application identifier algorithm at MAC layer for vehicular safety message dissemination over LTE VANET networks," Wireless Communications and Mobile Computing, vol. 2018, Article ID 6576287, 17 pages, 2018.

[4] L. Hu, Y. Xiao, and Z. Dai, "Beacon transmission rate allocation optimization under synchronized P-persistent repetition MAC protocol for platooning," Wireless Communications and Mobile Computing, vol. 2020, Article ID 8887134, 8 pages, 2020.

[5] M. Azizian, S. Cherkaoui, and A. S. Hafid, "A distributed cluster based transmission scheduling in VANET," in Proceedings of the 2016 IEEE International Conference on Communications (ICC), 2016, pp. 1-6, Kuala Lumpur, Malaysia, May 2016.

[6] V. Nguyen, O. T. T. Kim, C. Pham et al., "A survey on adaptive multi-channel MAC protocols in VANETs using Markov models," IEEE Access, vol. 6, pp. 16493-16514, 2018.

[7] Y. Bouchaala, P. Muhlethaler, O. Shagdar, and N. Achir, "Optimized spatial CSMA for VANETs: a comparative study using a simple stochastic model and simulation results," in Proceedings of the 2017 14th IEEE Annual Consumer Communications \& Networking Conference (CCNC), pp. 293-298, Las Vegas USA, January 2017.

[8] A. Bazzi, C. Campolo, B. M. Masini, A. Molinaro, A. Zanella, and A. O. Berthet, "Enhancing cooperative driving in IEEE 802.11 vehicular networks through full-duplex radios," IEEE Transactions on Wireless Communications, vol. 17, no. 4, pp. 2402-2416, 2018.

[9] A. T. Giang, A. Busson, A. Lambert, and D. Gruyer, "Spatial capacity of IEEE 802.11p-based VANET: models, simulations, and experimentations," IEEE Transactions on Vehicular Technology, vol. 65, no. 8, pp. 6454-6467, 2016.

[10] X. Cao, L. Liu, Y. Cheng, L. X. Cai, and C. Sun, "On optimal device-to-device resource allocation for minimizing end-toend delay in VANETs," IEEE Transactions on Vehicular Technology, vol. 65, no. 10, pp. 7905-7916, 2016.

[11] I. W. Group: IEEE Standard for Information Technology-Telecommunications and Information Exchange Between Systems-Local and Metropolitan Area
Networks-Specific Requirements-Part 11: Wireless LAN Medium Access Control (MAC) and Physical Layer (PHY) Specifications Amendment 6, pp. 1-2742, 2011.

[12] C.-Y. Chang, H.-C. Yen, and D.-J. Deng, "V2V QoS guaranteed channel access in IEEE 802.11p VANETs," IEEE Transactions on Dependable and Secure Computing, vol. 13, no. 1, pp. 5-17, 2016.

[13] Y. Wu and J. Zheng, "Modeling and analysis of the uplink local delay in MEC-based VANETs," IEEE Transactions on Vehicular Technology, vol. 69, no. 4, pp. 3538-3549, 2020.

[14] X. Jiang and D. H. C. Du, "PTMAC: a prediction-based TDMA MAC protocol for reducing packet collisions in VANET," IEEE Transactions on Vehicular Technology, vol. 65, no. 11, pp. 9209-9223, 2016.

[15] F. Lyu, H. Zhu, H. Zhou et al., "MoMAC: mobility-aware and collision-avoidance MAC for safety applications in VANETs," IEEE Transactions on Vehicular Technology, vol. 67, no. 11, pp. 10590-10602, 2018.

[16] D. N. M. Dang, H. N. Dang, V. Nguyen, Z. Htike, and C. S. Hong, "HER-MAC: a hybrid efficient and reliable MAC for vehicular ad hoc networks," in Proceedings of the IEEE 28th International Conference on Advanced Information Networking and Applications, pp. 186-193, Victoria, BC, May 2014.

[17] V. Nguyen, T. Z. Oo, P. Chuan, and C. S. Hong, "An efficient time slot acquisition on the hybrid TDMA/CSMA multichannel MAC in VANETs," IEEE Communications Letters, vol. 20, no. 5, pp. 970-973, 2016.

[18] S. Ucar, S. C. Ergen, and O. Ozkasap, "Multihop-cluster-based IEEE 802.11p and LTE hybrid architecture for VANET safety message dissemination," IEEE Transactions on Vehicular Technology, vol. 65, no. 4, pp. 2621-2636, 2016. 